Commentary/Block: Consciousness, accessibility, and the mesh

\title{
Conscious access overflows overt report
}

doi: $10.1017 /$ S0140525X07003044

Claire Sergent and Geraint Rees

Institute of Cognitive Neuroscience \& Wellcome Department of Imaging Neuroscience, Institute of Neurology, University College London, London WC1N3AR, United Kingdom.

csergent@fil.ion.ucl.ac.uk

http:// www.icn.ucl.ac.uk/Research-Groups/awareness-group

g.rees@fil.ion.ucl.ac.uk

http://www.fil.ion.ucl.ac.uk/ grees 
Abstract: Block proposes that phenomenal experience overflows conscious access. In contrast, we propose that conscious access overflows overt report. We argue that a theory of phenomenal experience cannot discard subjective report and that Block's examples of phenomenal "overflow" relate to two different types of perception. We propose that conscious access is more than simply readout of a preexisting phenomenal experience.

Visual presentation of a face can elicit strong activity in the fusiform gyrus even when a patient with neglect does not see it (Rees et al. 2000). Block's proposition that recurrent sensory activations constitute the core neural basis of phenomenal experience leads him to the following prediction:

[I]f the activations of the fusiform area [...] in the patient G.K. turn out to be recurrent activations, we would have evidence for phenomenal experience that the subject not only does not know about, but in these circumstances cannot know about. (target article, sect. 14, para. 13)

In other words, the experimenter could detect phenomenal experience in a patient's brain even if the patient denies having that experience! This contradicts the notion of phenomenal experience itself, which is, by definition, a subjective, firstperson experience. We suggest that any theory of phenomenal experience that discards subjective report as its fundamental measure is bound to lead to such contradictory predictions.

It is crucial to distinguish different forms of accessibility. In experiments using backward masking (Lau \& Passingham 2006; Vorberg et al. 2003), when subjects are pressed to guess which stimulus has been presented they can perform better than chance, even when they claim they have not seen it. In this case, as in classical "blindsight" (Weiskrantz 1997), there is a clear distinction between two types of report: objective report (accessibility in a direct sense, probably via automatic stimulusresponse routes) and subjective report (accessibility as defined in the term "conscious access"). We propose that this latter form of accessibility is intrinsic to what Block calls phenomenal consciousness.

In our view, the real challenge lies in designing tools to allow an objective, scientific measure of subjective report (Dennett 1992). Indeed, this is already an active area of study (Dienes \& Scott 2005; Macmillan \& Creelman 1991; Persaud et al. 2007; Sergent et al. 2005; Sergent \& Dehaene 2004; Weiskrantz 1997) and could in principle produce biomarkers of consciousness that allow report of phenomenal experience in the absence of overt report, via external brain imaging devices (Naccache 2006b; Nachev \& Husain 2007; Owen et al. 2006).

Block makes extensive use of partial-report paradigms (Landman et al. 2003; Sperling 1960) to support the argument that phenomenal experience overflows conscious accessibility. While an observer viewing such a letter array appears to "see it all," uncued report of the individual letters a short time later is poor. Nevertheless, a cue occurring after the array has been presented can lead to excellent recall of letter identity for the cued subset of the array. This excellent cued recall does not entail - as Block argues - that the earlier "seeing it all" experience therefore reflects phenomenal experience of all the letters at the same level of detail. Instead, we suggest that cued recall not only reflects readout of a fading information buffer, but it is a modification of perception through attention that does not differ much from classical sensory modulation by spatial attention (Ruff et al., in press). Although only the cued letters receive this additional attentional enhancement, the claim of "seeing it all" might still be based on access to lower-level attributes that are not explicitly probed by the experimenter - for example, presence of the uncued stimuli, rough recognition as "alphanumeric characters," or "Gestalt" properties of the scene gist. The apparent intuition lying behind phenomenal consciousness might therefore simply refer to access itself being much greater in some situations than the specific report required by the experimenter.
Strong sensory processing is consistently observed even when observers deny any form of experience of the stimulus. For example, the "attentional blink" describes impairment in reporting the second of two visual targets when they are separated by a short interval. Sergent and colleagues asked subjects to evaluate the visibility of a target word during the attentional blink on a continuous scale going from 0 to $100 \%$ visibility. If there were any form of experience of the target, even feeble or partial, they could acknowledge that by using intermediate visibility levels. However, missing a target corresponded to a subjective rating of zero, just as when no word was presented (Sergent \& Dehaene 2004). Nevertheless, these "zero-visibility" words elicited strong, long-lasting brain potentials up to 300 msecs after they had been presented (Sergent et al. 2005), a time scale at which local recurrent loops ought to have been established (Lamme \& Roelfsema 2000). Therefore, strong and elaborate processing within sensory areas is not sufficient for subjective experience.

In summary, we believe that what Block calls "phenomenal consciousness" actually reflects two different situations:

1. Genuinely unconscious stimuli that nevertheless receive strong sensory processing: this type of stimuli can influence our conscious mental life (Dehaene et al. 1998; Gaillard et al. 2006; Greenwald et al. 2003), but are not directly conscious.

2. Conscious stimuli that do not receive full attention and for which conscious access is partial to only certain attributes of the stimuli.

Finally, Block argues that activity in fronto-parietal areas does not reflect the core neural basis of consciousness and instead constitutes "read out" of the perceptual state represented in occipitotemporal areas. But the empirical evidence does not support a clear distinction between "perceptual" and "report" areas. For example, enhanced connectivity between fronto-parietal and visual cortices is associated with fluctuations in conscious contents during binocular rivalry (Lumer et al. 1998; Srinivasan et al. 1999) even when observers are not required to make any covert or overt reports of their experience (Lumer \& Rees 1999). Therefore, the evidence points to an active interaction among frontal, parietal, and sensory areas that altogether constitute conscious perception (Dehaene et al. 2003; 2006; Rees et al. 2002a).

In conclusion, we are far from understanding the complexity of the neural mechanisms that underlie our rich subjective experience of the external world, and Block's article provides a stimulating reminder of the path that lies ahead. But, in our view, the study of conscious access through careful assessment of subjective reports is the most positive and promising movement in that direction so far.

\section{ACKNOWLEDGMENTS}

This work is supported by the Wellcome Trust (for Geraint Rees), and the Fyssen Foundation and the Marie Curie European Program (for Claire Sergent). 